\title{
TINA said, There is no such thing as society
}

\begin{abstract}
Eine kleine Gedenk-Schrift an die geistig moralische Wende der wilden 1980er
Es gibt manche Gründe, sich ein stärkeres, verantwortlicheres Regieren über nationale Grenzen hinaus zu wünschen. Aber der Blick in die zweite Hälfte des 20. Jahrhunderts gibt auch Grund zur Skepsis - z. B. besteht mehr Europa, als wir wahrhaben wollen, nur wieder das Falsche?
\end{abstract}

$\mathrm{E}$ s gibt also keine Alternative, weil es eben keine Gesellschaft gibt - und wer Margaret Thatcher ein wenig kannte, weiß auch, dass das, was sie 1987 im Interview mit einer Frauenzeitschrift sagte ${ }^{1}$, keineswegs eine bedauernde Feststellung war.

\section{Es war einmal ...}

Heute wird nun genau das beklagt, was sie seinerzeit feststellte. Wenn heute überhaupt etwas Geschichtliches bei dem Hinweis auftaucht, ist es ein allgemeines Bedauern, dass der Egoismus (sic!) immer schlimmer wird, die "Gier“ gar Ursache der Krise war und dann - ja erst dann - der Neoliberalismus die Reste von Gesellschaft abgebaut habe: Sozialstaatsabbau, Austerität, Kapitalfreundlichkeit oder gar -hörigkeit sind dann schnell bei der Hand und wenn eine_r etwas reisen muss, so kommt er schnell zum Überdruss, denn wohin man auch immer etwa in Europa kommt, ist es besonders schlimm: „Oh, unser Pflegenotstand“ - „Il numero sempre cre-

Prof. Dr. phil. Peter Herrmann', 2
'Berlin, Deutschland
2University of Eastern Finland (UEF) including Kuopio University
Hospital, Kuopio, Finnland
*1955, Sozialphilosoph
herrmann@esosc.eu

Zusammenfassung Alles wird heute unter dem Begriff Neoliberalismus abgetan - und dies scheint ein mehr oder weniger junges Maßnahmenbündel. Übersehen wird aber, dass das, was heute so bezeichnet wird, eine lange Vorgeschichte hat und wenig mit Maßnahmen zu tun hat, dafür aber sehr viel mit der Durchsetzung einer auf die Spitze getriebenen Modernisierung: es ging immer um Individualisierung als spezifische Vergesellschaftungsform - nur wenn man dieses Oxymoron denk-zulässt, kann man verstehen, wie tief die Wunden heute gegraben werden - wahrhaft nachhaltige Politik.

Schlüsselwörter Wirtschaft und Gesellschaft, Hegemonie, Ent-Solidarisierung, 1968er scente di disoccupati“ - „Qu'est-ce que l'état fait pour s'occuper des enfants - Accommodation?" - „Who can still go for that? "...

Dabei weiß doch jeder, dass es „dort“ überall so schlimm nicht ist, denn besonders dramatisch ist es eben doch nur im eigenen Land. Ironie beiseite, der Drang, Dinge auf Punkt, Ort und Zeit zu bringen, auf das Hier und Jetzt zu reduzieren, ist stark - und doch ist genau dies auch Teil jener Politik, die sich bereits Ende der 1970er Jahre an einen Gesellschaftsumbau machte: es war der Punkt eines methodologischen Nationalismus: mit der Nachricht „Wir sind die Ärmsten“ wurde der Grundstein für besonderen Fleiß gelegt, aber ebenso für die besondere Hinnahme-Bereitschaft für politische Änderungen. Im Grunde wirkt eben auch hier die vom „Urkanzler Bismarck“ verfochtene Politik von Zuckerbrot und Peitsche. Es ist allerdings mehr als bemerkenswert, dass die Bismarck'sche Sozialversicherung letztlich maßgeblich auch wegen der Bemühungen des seinerzeitigen Handelsministers durchgesetzt wurde. Zugespitzt: Bismarck hielt die Peitsche in der Hand und die Handelsinteressierten verteilten Zuckerstückchen.

...und nun wird's wahr...

Es mag Zufall sein, dass die Neuauflage des Films Invasion of the Body Snatchers genau diese Zeit einleitete laut imdb-Datenbank geht es darum, dass „In San Francisco, a group of people discover the human race is being replaced one by one, with clones devoid of emotion." (https://www.imdb.com/title/tt0077745/; 23.02.19).

Leinwand frei also für ein spektakuläres Spiel - allerdings folgte es dann nicht nur auf der Leinwand, sondern auf der gesellschaftlichen Bühne, auch wenn es diese ja angeblich gar nicht gab.

\section{Es darf nicht sein}

Ein Blick auf die Gemengelage jener Wendejahre muss ein wenig verwundern, denn der erste Eindruck wurde 


\section{Durchblick: „Sie verlassen den sozialen Sektor“}

\section{Übersicht: 1968 und der paradoxe Nachhall}

Nur eine Kleinigkeit

...relative Stabilisierung der Nach-68er-Erfolgsepoche, wenngleich als labiles Spannungsfeld (siehe https://www.buko.info/http/kongress/buko31/ deutsch/groups/68_zeitstrahl.pdf; 23.02.19)

\section{Öffnung der Ausgangstür}

1979: Regierungsübernahme durch Margaret Thatcher; Übernahme der Regierung von Labour (Leonard James Callaghan)

Seit 1980 in verschiedenen Reden Kohls die Forderung nach einer „geistig-moralischen Wende“, „geistigen Erneuerung “...

September 1982: Landtagswahl in Hessen mit den ersten Landtagsmandaten für die Grünen

Oktober 1982: Misstrauensvotum gegen die Regierung Schmidt und Regierungserklärung

März 1983: Bundestagswahl, mit erheblichen Gewinnen für die CDU/CSU und dem erstmaligen Einzug der Grünen in den Bundestag

\section{Erste Evakuierung}

1982 eine erste Ablehnung der EU- „Programme zur Armutsbekämpfung “

1995 Urteil des EuGH mit der Bekräftigung, dass die EU tatsächlich keine sozialpolitische Kompetenz hat

1997: EU-Vertragsdefinition: Armutsbekämpfung ist Arbeitsmarktpolitik und im Amsterdamer Vertragswerk verankert.

\section{Versperren des Rückweges}

2006 Richtlinie 2006/123/EG des Europäischen Parlaments und des Rates vom 12. Dezember 2006 über Dienstleistungen im Binnenmarkt ${ }^{2}$

\section{Epilog}

2012 - laut Wikipedia: „Der gestürzte Helmut Schmidt sagte 2012 dazu rückblickend in einem Interview, dass es gar keine Wende gegeben habe, sondern die sozialliberale Politik fortgeführt wurde und ,nur das Personal ausgetauscht' worden sei.“ (https:// de.wikipedia.org/wiki/Geistig-moralische_Wende; 23.02.19)

durchaus als positiv dargestellt: so wie die Studierenden sich gegen den Muff von tausend Jahren gewandt hatten, stemmten sich die neuen Ordnungsstifter gegen den Staub, der dort in weniger als einem halben Jahrhundert aufgewirbelt worden war. Diese Formulierung ist aber gleich zu berichtigen:
- richtiger ist wohl, von neuen Stiftern der alten Ordnung zu sprechen, denn zumindest waren einige der alten Stifter verstorben oder wurden, wie etwa AltNazi Kiesinger, vertrieben.

- Ebenso ging es darum, dass der aufgewirbelte Staub ja gerade auch jener Staub war, der im Grunde noch von den Vätern der neuen Stifter hinterlassen war von Vietnam bis Pinochet-Hayeks Chile (s. dazu Farrant 2012).

Erfolg und nachhaltige Wirkung der '68er stehen hier nicht zur Debatte - mit aller erforderlichen Vorsicht aber lässt sich wohl von einer relativ erfolgreichen Durchsetzung des Sozialstaatsgedankens sprechen: auch hier spielten sicher Peitschenhiebe eine Rolle - in Form eines Bürgerkrieges an mehreren Fronten; ${ }^{3}$ aber doch schienen die Zuckerbrote ausreichend groß, um zumindest eine vorübergehende Beruhigung sicherzustellen. Für Deutschland muss zumindest gesagt werden: was sich da als sozialer Sektor darstellte, war nicht so ganz problemlos - zumindest drei Moment sollten nicht vergessen werden:

1. Das zweite deutsche „Sozialwirtschaftswunder“ war vor allem Resultat einer recht aggressiven Exportorientierung - und damit grub es sich im Grunde seine eigene Grube

2. Nicht zuletzt gelang es, einen nicht geringen Teil der Probleme auszulagern oder aber zu verstecken - erst später sollte dies wirklich virulent werden: Armut gab es nicht - sie wurde amtlich wegdefiniert, indem Sozialhilfebezug als bekämpfte Armut eingestuft wurde.

3. Auch profilierte sich Deutschland - vor allem zusammen mit Dänemark, den Niederlanden und dem Vereinigten Königreich - in der 1972 zögerlichen Europäischen Sozialordnungspolitik, die cum grano salis wohl nur im Rückblick ihr wahres Wesen zeigt, und man muss wohl (zumindest einigen unter) den „Machern“ zugestehen, dass keiner die Ausgangstür geöffnet hat, aber doch plötzlich alle in einer Art sozialem Abseits standen.

\section{Was lange währt, wird längst nicht gut}

Die „freie, nicht erfolgsgebundene“ Orientierung als Regelaufgabe (s. Scherr in dieser Ausgabe) fand eine symbolische Ergänzung durch die Programm- und Projektpolitik, die in der Tat durchaus teils spannende ErÖffnungen bot (siehe etwa Herrmann 2000 und 2009) - das Traurig-Spannende daran war aber eben genau die falsche Zurückhaltung (Herrmann 1995): Subsidiarität wurde von Professionsvertretern als Möglichkeit der Bestandssicherung gesehen und auch genutzt, neue 
Erfahrungsräume zu erschließen, zugleich gab es aber keine Kraft, die dies als Grundlage für eine relevante Politik auf der EU-Ebene nutzte und endlich EU-Verantwortung einforderte - für die Projekte gab es dort keine Einflussmöglichkeiten, die Profession war mit der Tagesarbeit ausgelastet, den Kollegen der akademischen Welt fehlte meist der Zugang, da es kaum relevante Projektausschreibungen gab und die Ausnahmen das Einlassen auf bürokratischen Aufwand und den Mut zur Fremdsprache abverlangten. Die Wohlfahrtsverbände waren überfordert, denn das EU-Tagesgeschäft verlangte Vieles und Anderes: etwa die europa-steuer-rechtliche Behandlung von Briefmarken mit Wohlfahrtszuschlag. Von Regierungsvertretern wurden diese Aktivitäten eher als Entschuldigung für Enthaltsamkeit interpretiert, denn schließlich gab es keine EU-Kompetenz.

Grenztechnisch war es eine Wanderung im terra nullius: dem Land, das keinem gehört, was damit zum Land auch der Besitzlosen wurde. Eine innere Landnahme fand dann stückweise dadurch statt, dass weitere Aktivitäten immer strikter an die „Kernaufgaben der EU“ gebunden wurden. Im Klartext: wo ein Großteil des Sozialen schon draußen stand, wurden die Grenzbäume heruntergelassen: Wettbewerb war nun das magische Wort - keineswegs als Ausschluss des Sozialen präsentiert. Es sollte alles noch besser werden, nun auch endlich wirklich EUropäisch, etwa durch freien Dienstleistungsverkehr (Herrmann 2011). Das kann doch nicht als Verlassen des sozialen Sektors gesehen werden? Vielmehr wurde so die volle Anerkennung des Sozialen erreicht: Sozialdienstleister als gleichberechtigte Wirtschaftspartner, etwa neben der Deutschen Bank, HochTief oder eben auch Flixbus.

\section{Wer einmal in den Blechnapf schaut...}

So war es gleichsam visionär: Kohl und Thatcher bereiteten mit ihren Slogans gleichsam die Hauptzutaten für eine „alchimistische Zirkel-Suppe“, die den Sozialraum, wie wir ihn kannten, systematisch umdefinierten und Teile der Profession tatsächlich im Kreis laufen ließ. Im Grunde haben wir wieder eine Konstellation, bei der Handels- und allgemeine Sozialordnungspolitik zusammenfinden müssen. Die EU-Festungspolitik des globalen Handels geht die üblichen Querpfade einer nur einseitig verschlossenen Festungsmauer. Zudem geht es gegenwärtig auch darum, dass sich weitgehende Strukturverschiebungen ergeben - Stichworte sind Digitalisierung, Dienstleistungs-, Handels- und Finanzkapitalismus, zumindest in den westlichen Zentren - und schließlich zugleich auch die sog. Brasilianisierung. Das Entscheidende ist, dass auf diese Weise ein „Klima“ geschaffen wird, das viel durchgreifender wirkt als das, was immer als Neoliberalismus bezeichnet wird. Das Verlassen des sozialen Raums wurde geschickter vorangetrieben, als jegliche Kürzungs-, Spar- und Umbaupolitiken hätten erreichen können: Es handelt sich gleichsam um eine Art „innere Emigration“ - fast genau schreiben wir das vierzigste Jahr dieser Gegenrevolution: Angetreten war sie gegen die dämonisierten Sozialisten, vorab klarstellend: „Our aim is not just to remove our uniquely incompetent Government from office - it is to destroy the socialist fallacies - indeed the whole fallacy of socialism - that the Labour Party exists to spread“. ${ }^{4}$

Das war schnell als die eigentliche Kampfaufgabe sichtbar übersetzt, weit darüber hinausgehend, den kurzfristigen die Interessen der Wirtschaftsbosse zu dienen. In der Tat wurde der Blick auf die Wirtschaft viel grundsätzlicher gesehen - Kohl etwa erwies sich als eine Art Gramscianer und stellte in der Regierungserklärung fest: „Das schließliche Schicksal der Marktwirtschaft entscheidet sich - jenseits von Angebot und Nachfrage". 5

Auch hier ging es schließlich nicht schlicht um die realen Probleme ${ }^{6}$, sondern auch um die gemachten bzw. genauer, diejenigen, die angeblich nicht bestanden: „Die neue Armut ist eine Erfindung des sozialistischen Jet Sets.“ (STERN, 24. Juli 1986).

Es lassen sich unendliche Aussagen anführen - bezeichnend ist, dass es sich oft um Negativ-Aussagen handelt; Kritiken, die gleichsam auch negativ beschrieben, was die Konservativen eben nicht sein wollten, programmatischer Hauptpunkt war: sozial und verantwortlich - bereits $1949^{7}$ - plädierte Thatcher für das Ideal der später durch Angela Merkel bekannt gewordenen „schwäbischen Hausfrau“. Demzufolge haben auch Staatshaushalte das Ziel, keine Schulden zu machen.

Vor allem aber wollten sie keine Sozialisten sein - bemerkenswert, wenn man bedenkt, dass der Vorgänger dieser a-sozialen Ära, Schmidt, sagte, dass es eh keine Wende gegeben habe (siehe Kasten); und ebenso bemerkenswert, dass dann der „dritte Weg“, der später angeblich mit Tony Blair im VK (1997) und Gerhard Schroeder in der Bundesrepublik (1998) seinerzeit kurz vor der Wahl - in einem Artikel in der Financial Times (internationale Ausgabe; passim) herbeigesehnt wurde - mit dem Tenor, dass ein Wechsel erforderlich sei, um die Weiterführung des konservativen Weges zu sichern. Im Grunde war das „Meister“werk geschafft: der soziale Raum war verlassen, an die Tür wurde ein Schild gehängt: „Vorsicht, nicht zurückschauen! Giftablagerungen!“ Die Grundproblematik, die Sibel Kalaycioglu am Beispiel der syrischen Flüchtlinge in der Türkei in diesem Heft betrachtet, zusammengefasst in der Aussage „Humanitäre Heuchelei versus Menschen- 


\section{Durchblick: „Sie verlassen den sozialen Sektor“}

rechtspolitik", gilt cum grano salis bis tief in den EUKern hinein.

Und neue Würgeträger wurden zielgerecht ausgebil$\operatorname{det}$ (s. Bohnenberger in diesem Heft) - sie sollten sich u. a. auf Privatdienste, Konkurrenz, Spaltung, und als Eifer verbrämten Stress für „Sozialkunden“ und „Anbieter" einstellen, und nun endlich die guten Wünsche von TINA zu akzeptieren. Vieles haben diese „Sozialisten " übersehen, u. a. dass sie einer Alternative falscher Demagogen den Weg bereitet haben (siehe Butterwegge in diesem Heft).

TINA: There is no alternative.

Funding. Open access funding provided by University of Eastern Finland (UEF) including Kuopio University Hospital.

Open Access. Dieser Artikel wird unter der Creative Commons Namensnennung 4.0 International Lizenz (http://creativecommons.org/ licenses/by/4.0/deed.de) veröffentlicht, welche die Nutzung, Vervielfältigung, Bearbeitung, Verbreitung und Wiedergabe in jeglichem Medium und Format erlaubt, sofern Sie den/die ursprünglichen Autor(en) und die Quelle ordnungsgemäß nennen, einen Link zur Creative Commons Lizenz beifügen und angeben, ob Änderungen vorgenommen wurden.

1. Thatcher, Margaret, interviewed by Douglas Keay (1987, September 23). Interview for Woman's Own; Thatcher Archive (THCR 5/2/262): COI transcript; https://www.margaretthatcher.org/document/106689; 17/06/18.

2. OJ L 376, 27.12.2006, p. 36-68; http://data.europa.eu/eli/ dir/2006/123/oj; 24.02.19.

3. Streikniederschlagungen, antigewerkschaftliche Aktivitäten, Rechtsbeugungen, Berufsverbote, Krieg mit der/gegen die RAF.

4. Speech to Junior Carlton Club Political Council (4 May 1976). https://www.margaretthatcher.org/document/103017.

5. Auszug aus der Regierungserklärung: https:// www.1000dokumente.de/pdfldok_0144_koh_de.pdf.

6. Zugegebenermaßen nennt Kohl in der Regierungserklärung durchaus mit einer gewissen Systematik einige der Kernprobleme, auch die, die nicht nur offensichtliche Tatbestände betreffen, sondern auch solche, die eher techno-politischen Charakter haben (Geld-und Haushaltspolitik etc.).

7. Speech at her adoption meeting as Conservative candidate for Dartford (28 February 1949); https://www.margaretthatcher.org/ document/100821; 24.02.19.

\section{Literatur}

Farrant, A., et al. (2012). Preventing the 'abuses' of democracy: Hayek, the 'military usurper' and transitional dictatorship in Chile? American Journal of Economics and Sociology, 71(3), 513-538.

Herrmann, P. (1995). Subsidiariät und die falsche Zurückhaltung oder: Über den Sinn europäischer Armutsprogramme. Nachrichtendienst des Deutschen Vereins Für öffentliche und private Fürsorge, 76(2), 79-86.

Herrmann, P. (2000). Sozialarbeit und Europäische Integration - Gefahren, den linken mit dem rechten Schuh zu verwechseln. Neue Praxis, 30(6), 601-607.
Herrmann, P. (2009). Die Europäische Union als Programmgesellschaft. Das Europäische Gesellschaftsmodell, die Sozialpolitik und der Dritte Sektor. Studien zu vergleichender Sozialpädagogik und internationaler Sozialarbeit und Sozialpolitik, Bd. I. Bremen: Europäischer Hochschulverlag.

Herrmann, P. (2011). The end of social services? Economisation and Managerialism. Bremen: Europäischer Hochschulverlag. 